\title{
La politique des émotions suscitées par la formalisation esthétique de la photographie de Sebastião Salgado
}

Katia MACHADO, Université de la Sorbonne Nouvelle (Paris 3)

\section{Introduction}

Pour décrédibiliser la conception du photoreportage de Sebastião Salgado, le principal argument des critiques opposants est que les émotions perturbent la faculté de réflexion du spectateur. Ils considèrent également que sous l'effet des émotions les spectateurs semblent être anesthésiés devant la représentation de la souffrance des autres. En raison des spécificités du procédé de communication de l'image photographique, le soupçon de manipulation de la part de l'énonciateur n'est pas difficile à entrevoir. L'appréhension de ce qui est montré dans une photographie étant quasi immédiate, ce type d'image-indice s'avère être une source d'émotions directes. Comme le souligne Roland Barthes, la photographie peut nous « regarder droit dans les yeux », tandis que «dans le film, personne ne me regarde jamais : c'est interdit - par la fiction » (172). En outre, la photographie parle sans dire. Elle n'a donc pas de moyens de s'auto-expliquer afin de préciser le sens de ce qui est énoncé. La combinaison de ces deux facteurs suggère une certaine vulnérabilité du spectateur à se laisser manipuler. De sorte que ce n'est pas seulement en raison des trucages dans le procédé technique ou de la pratique de mise en scène que l'on met en cause la légitimité d'une énonciation photographique, mais aussi lorsqu'elle fait appel aux sentiments, notamment si le photographe se propose de dénoncer la réalité sociale que sa photographie énonce comme c'est le cas chez Sebastião Salgado. Néanmoins, «l'image, même photographique, ne peut valoir que par l'émotion qu'elle suscite et jamais pour la vérité qu'elle prouve » (Mondzain, «Commerce », 230). Ainsi, dans cet article nous tâcherons de montrer le rôle des émotions dans le processus de raisonnement du spectateur d'images, leur influence dans le comportement éthique des êtres humains, et en quoi l'éveil des émotions sociales positives suscitées devant les représentations photographiques des personnes en détresse sociale peuvent s'avérer être un remède contre notre habituelle indifférence à la souffrance du monde.

\section{Quel intérêt sociologique de la question des émotions éprouvées devant les images qui circulent dans le tissu social ?}

L'un des intérêts réside dans le fait que ces images socialisent. Elles sont exposées au 
regard d'une multitude de personnes qui pour la plupart ne se sont jamais vues, jamais connues, mais faisant l'expérience de « sentir ensemble » partagent une sensibilité commune. Les émotions suscitées ont ainsi une raison d'être sociale car, même si elles peuvent varier en fonction de l'individualité de chaque individu, elles sont, néanmoins, des subjectivités intersubjectives. «Personne ne pourra jamais se vanter de savoir ce que l'autre voit ou ressent devant le spectacle du monde, et cependant une communauté ne peut se maintenir dans le partage de ce monde qu'en se donnant des moyens d'y constituer des réseaux de signes qui circulent entre les corps et produisent une sociabilité politique des émotions » (180).

La question de savoir quel type d'émotion une image suscite, n'est donc pas sans importance. Elle est même fondamentale, voire cruciale, lorsqu'il s'agit d'interroger les images qui représentent des ensembles d'individus socialement opprimés car ce ne sont pas seulement les émotions positives qui font objet de socialisation, et ce n'est pas toujours non plus un discours visuel qui a pour finalité de produire des effets contribuant à changer pour le meilleur la condition de vie des personnes représentées. Mais ce qui est certain c'est que les émotions, une fois socialisées, font que les gens éprouvent ensemble des sentiments, les ressentent ensemble pour le meilleur mais aussi pour le pire. Et le problème le plus important qui se pose en tout cela est que l'expérience du sentir ensemble procurée par les images se révèle comme une modalité de l'exercice du pouvoir. Car, comme souligné par Marie-José Mondzain, «le visible nous affecte en tant qu'il a affaire avec la puissance du désir et qu'il nous met en demeure de trouver les moyens d'aimer ou de haïr ensemble » («Image », 22). Pour sa part, «le pouvoir veut toujours contrôler l'amour et la haine, et dans la mesure où l'émotion visuelle a affaire à ces passions-là, le dispositif qui montre, la forme choisie pour montrer, la place donnée à la voix, le risque pris dans un cadrage, dans un montage, sont autant de gestes politiques où s'engage le destin du spectateur dans sa liberté même » (5657).

L'autre intérêt sociologique du rapport entre image et émotion repose sur la question de l'influence des émotions sur la capacité de réflexion du spectateur. En tant que support de communication, l'image ne trouble pas de soi même la faculté de réflexion du spectateur. $\mathrm{Au}$ contraire, elle la favorise. Du point de vue du fonctionnement psychique des individus, Serge Tisseron précise que : «pour commencer à penser le monde, il faut établir entre lui et nous un écran de projection qui est aussi un écran de protection. L'image est cet écran » (52). Mais qu'en est-il du rôle des émotions suscitées par les images? Les points de vue convergent pour signaler que les émotions participent du processus de réception de l'image. Mais pourtant, les opinions sur le caractère «bon » ou «mauvais » des effets que cette participation produit sur 
la faculté de raisonnement du spectateur s'avèrent assez divergentes. Tantôt on les considère sans pouvoir en ce sens que les émotions éprouvées devant une image seraient vécues comme dénuées de signification (Aumont). Tantôt comme une modalité de symbolisation de l'assimilation psychique contribuant à la construction de la signification de l'image (Tisseron). D'autres fois encore, on les estime catégoriquement comme une intervention négative. C'est le cas des critiques opposés à l'approche photographique de Salgado, ce que l'on peut comprendre en lisant "entre les lignes" de l'argumentation d'Ingrid Sischy et de Jean-François Chevrier. Michel Guerrin énonce sans ambages : «l'émotion occulte la réflexion » (Le Monde, 11/04/2000).

\section{2. Émotion et raison : incompatibles ?}

Les résultats obtenus dans les investigations menées par Antonio Damasio avec son équipe à l'Université de l'Jowa sur le fonctionnement des émotions et des sentiments apportent des réponses concluantes à cette question. Pendant longtemps l'idée dominante fut que les émotions n'avaient pas une base matérielle comme cause d'origine. Elles étaient traitées comme une affaire insondable de l'esprit humain. Cette croyance était nourrie par la notion, largement partagée dans le milieu des neurosciences selon laquelle la raison et les émotions relevaient des circuits neuronaux indépendants.

Ayant appris dans son plus jeune âge «que les émotions et la raison ne pouvaient pas plus se conjuguer que l'eau et l'huile », Damasio a cru lui-même pendant longtemps que pour prendre des sages décisions on ne peut pas être sous l'influence des émotions («Descartes», 7). Puis un tournant s'est produit. Inspiré par ses années d'observations cliniques et de sa lecture de Spinoza, Damasio pose comme hypothèse de travail qu'au lieu de déranger le processus du raisonnement, les émotions participent à sa réussite.

Du point de vue neurobiologique, les émotions et les sentiments sont, d'après Damasio, deux facettes d'un seul et même phénomène: les mécanismes qui forment la base de la régulation de la vie. À la différence que «les émotions se manifestent sur le théâtre du corps ; les sentiments sur celui de l'esprit» («Spinoza », 34). Les émotions sont des actions ou des mouvements. Les sentiments sont, à leur tour, des perceptions. Ils déclenchent en même temps la perception de l'émotion, et la perception de la cause de cette émotion. Cela nous conduit à savoir pourquoi nous avons telle ou telle émotion, débouchant ainsi sur le libre arbitre. Il s'agit donc d'une réaction médiatisée par la raison. Et c'est cette médiation qui permet de comprendre le sens de l'émotion. Tandis que l'émotion est de l'ordre du commandement, le sentiment est de l'ordre du jugement. Autant dire que ce dernier est le 
commencement de la conscience.

Diverses expériences ont permis de constater qu'il existe une interconnexion entre les systèmes cérébraux qui contrôlent le fonctionnement de la raison et ceux de l'émotion, et que cette dernière assiste la mise en œuvre efficace de la première. Ainsi, l'investigation d'une population de patients souffrant à la fois de défauts de prise de décision et de troubles de l'émotion a montré que leurs défauts de raisonnement sont dus à l'endommagement de la transmission de signaux liés à l'émotion. Ce qui amène Damasio à conclure « qu'émotion et sentiment non seulement jouent dans le processus du raisonnement, mais jouent même de façon indispensable » (154).

Ces apports scientifiques ne nous disent pas pourquoi telle image provoque telle réaction chez telle personne mais ils mettent en évidence que l'émotion n'occulte pas la réflexion. Mais, comme c'est, en effet, la faculté du raisonnement qui fournit un discernement logique entre le bon et le mauvais, même une fois démonté l'argument du "dérangement", on peut toujours objecter que dans les jugements qui portent sur des problèmes sociaux, l'appel aux émotions manque d'un objectif justifiable. Tel est le cas de l'argument utilisé par Sischy qui reproche à l'approche photographique de Salgado un manque de recul émotionnel tout comme le fait Walker Evans.

L'aspect singulier le plus caractéristique de la photographie d'Evans, écrit Sischy, c'est sa pureté, voire son purisme. C'est la photographie «directe» non seulement sur le plan technique mais aussi dans la rigoureuse droiture de sa manière de regarder... La vision est clinique. Evans est un docteur visuel, un diagnostiqueur plutôt qu'un spécialiste. Mais il est aussi le médecin de famille, réservé et détaché, devant qui même les personnes âgées ou malades non plus l'honte de se révéler eux-mêmes (The New Yorker, 09/09/1991).

En effet, pour faire comprendre ce qui est «bon» ou «mauvais » ainsi que pour informer sur l'existence des souffrances sociales, il n'est pas besoin de faire intervenir les émotions. Le recours au raisonnement logique suffit largement. De ce point de vue, il n'y a pas de contestation à faire à l'objection de Sischy. Mais il se trouve que pour Salgado, il ne s'agit pas simplement de faire comprendre ou d'informer. Son propos est de faire en sorte que ses images puissent motiver le spectateur à agir d'une façon quelconque en vue de combattre les causes productrices des souffrances représentées. Car la conviction de Salgado est que : « la photographie peut aider à changer le comportement, à changer le monde » (Jornal de Fotografia, 09/06/1995).

C'est sans doute parce que l'homme est un animal rationnel qu'il possède une conscience éthique, mais cela n'est pas forcément un facteur qui induit à un comportement 
bienveillant. Par la suite, on verra quelques raisons qui justifient le caractère même nécessaire de l'appel aux émotions sociales positives dans les interrelations humaines où il existe une forme quelconque de revendication de reconnaissance d'autrui. On découvrira ainsi que la raison toute seule n'est capable de mobiliser quoi que ce soit.

\section{Des bonnes raisons pour faire appel aux émotions}

Pour Spinoza les pouvoirs de la raison sont limités. La raison a le pouvoir de dire ce qu'il faut faire, mais n'a pas la force de déterminer le comportement. Pour cela il faut qu'elle même devienne un affect. Car il ne suffit pas de savoir ce qui est bon et ce qui est mal, il faut aussi savoir comment faire pour que le comportement soit spontanément le bon. On doit donc, d'une certaine façon, incorporer et rendre affectif ce que la raison nous enseigne.

Cette limitation est confirmée dans les expériences où Damasio cherche à répondre à la question du rôle des sentiments dans le comportement social des êtres humains. Des patients ayant un historique de lésion dans le cortex pré-frontal, par exemple, connaissent très bien les règles morales et les lois qu'ils étaient capables d'appliquer avant la lésion. Ce qui montre que leur capacité de raisonner intelligemment n'a pas été affectée. Mais après leur lésion ils font des erreurs systématiques dont ils sont capables de reconnaître le tort, mais sur lesquelles ils ne sont plus capables de revenir. Tout se passe comme si sans le sentiment de l'émotion on n'était plus capable de réaliser cette donnée éthique.

C'est pour une bonne partie grâce aux compétences émotionnelles, et non uniquement à la faculté de la raison, que la vie en société fondée sur des principes coopératifs a été une réalité possible. Les facultés émotionnelles jouent un rôle d'extrême importance dans la compréhension des significations existentielles que les situations peuvent avoir pour les individus. On sait catégoriser dans un répertoire cognitif telle ou telle conduite comme éthiquement bonne ou mauvaise, parce que chacune de nos conduites produit des effets positifs ou négatifs, identifiés comme tels justement parce qu'ils nous font éprouver respectivement des sentiments positifs ou négatifs.

Prenant appui sur les apports de la théorie de la psychologie du développement, Axel Honneth montre, d'un point de vue sociologique, que dans le comportement social de l'homme il y a «un primat à la fois génétique et catégoriel de la reconnaissance sur la connaissance »(71). Selon lui, il existe non seulement un caractère inné de l'expression émotive chez les humains, mais aussi une dépendance directe du bon fonctionnement de la faculté émotive dans le développement de la faculté cognitive. Position qui s'oppose diamétralement avec la sociologie des émotions de Maurice Halbwachs qui postule que 
l'expression émotive n'est pas une faculté naturelle, innée ou héréditaire, et, donc, ne provient pas de la constitution organique de l'espèce.

Les théories issues des recherches menées dans le domaine de la psychologie du développement convergent au même principe : «L'éveil des aptitudes du jeune enfant à penser et à interagir doit être conçu comme un processus qui prend place au cœur même du mécanisme par lequel ce même enfant adopte la perspective de l'autre personne qui lui fait face » (Honneth 53). L'adoption de la perspective d'une seconde personne, en l'occurrence la personne privilégiée de son entourage, fournit à l'enfant une instance de correction que lui permet de percevoir le monde qui l'entoure de manière impersonnelle et objective. C'est à travers cette relation triangulaire que le jeune enfant acquiert des compétences cognitives. Mais une erreur largement répandue consiste à la décrire comme un espace qui, pour l'essentiel, est dépourvu d'émotions. Les découvertes faites dans des études du cas des enfants autistes montrent néanmoins que ce qui fait défaut au développement de leur faculté cognitive c'est leur incapacité de réactivité sur le plan émotionnel. Pour adopter la perspective de la seconde personne, il faut d'abord que l'enfant la reconnaisse au sens émotionnel du terme. Seulement ainsi, il sera attiré et motivé par sa présence «d'une manière telle qu'il puisse saisir les changements d'attitude que manifestent cette personne en s'y intéressant » (57).

Dans le cas d'un enfant « normal » ${ }^{1}$ c'est l'identification émotionnelle avec autrui qui lui fait adopter sa perspective conduisant ainsi au développement de la pensée symbolique. Quant à l'enfant autiste :

Il ne voit pas, ou plutôt il ne sent pas que les expressions du visage, les mouvements et les gestes de communication expriment des attitudes. Il est aveugle au contenu expressif et mental de ces expressions, ou bien, comme on peut dire aussi, il est aveugle à leur signification. Si le jeune enfant autiste est ainsi «aveugle spirituellement», ce n'est pas en raison d'un déficit cognitif ; s'il est aveugle spirituellement, c'est d'abord parce qu'il est aveugle émotionnellement (58).

C'est donc parce qu'on naît émotionnellement compétent qu'on peut développer des compétences cognitives et, par conséquent, qu'on acquiert une conscience morale. Le paradoxe se trouve dans le fait que l'expression émotive devient au cours de la vie, pour une part, le produit de l'apprentissage, comme le montre clairement Halbwachs. Toutefois, la démonstration du primat de la reconnaissance sur la connaissance est une autre bonne justification pour faire intervenir les émotions dans les discours qui portent sur la question des personnes affectées par les souffrances sociales. 
En outre, les investigations de Gehard Roth révèlent que les émotions donnent l'impulsion pour agir. La réflexion ne sera traduite en action que si quelque chose d'émotionnel, conscient ou inconscient, s'y intègre :

La raison toute seule, on peut le démontrer dans l'anatomie et la physiologie cérébrales, ne peut mobiliser rien du tout. L'appareil qui produit notre raison dans le cerveau a été construit évolutivement d'une telle manière que lui-même tout seul ne mobilise pas quoi que ce soit. On a besoin d'une instance pour pouvoir réfléchir sur ce qu'on doit faire avant de le faire - celle-ci est la raison. Mais elle s'arrête là, sans donner l'impulsion pour agir. (Spiegel Wissen, 1/2009)

Les émotions étant le moteur propulseur de l'action et déterminant la qualité de celle-ci, il semble légitime de s'intéresser à la question du type de sentiment stimulé dans les représentations photographiques du monde social. L'existence des souffrances sociales, montrées dans les images de Salgado, est bien connue. Le système de communication d'informations du mass media nous tient au courant de tout ce qui se passe dans le monde. Mais, comme le souligne Régis Debray, « restait à le sentir, à le saisir hic et nunc, en direct, sans phrases », car « la médiatisation, paradoxalement, escamote à la longue ce qu'elle croit nous communiquer » (Marianne, 22/03/1999). On remarque en effet que cela est d'autant plus vrai quand la médiatisation offre une "vision clinique" du malheur d'autrui, et s'arrête là. Car la mobilisation des consciences demande toujours la stimulation prescrite par Damasio et Roth. Et c'est justement dans sa capacité à combler ce manque habituel que Debray indique l'un des aspects efficaces de l'approche photographique de Salgado : «Ces histoires en noir et blanc font émerger, à la place d'une humanité confuse et vague, faite, pour nous, de chiffres, de noms exotiques et incompréhensibles, de dépêches ou de flashs -, une humanitésoeur, avec des yeux, un corps, une âme » (Marianne, 22/03/1999).

On sait que les émotions sociales positives favorisent les comportements bienveillants à l'égard d'autrui et que les émotions sociales négatives opèrent dans le sens contraire. Comment peut-on alors attendre des représentations visuelles de la détresse sociale qui ne suscitent pas d'émotions sociales positives qu'elles puissent motiver à agir de manière bienveillante à l'égard des personnes représentées ? Cette question n'aurait pas beaucoup d'importance si des paradoxes n'habitaient pas la nature humaine.

\section{4. À cause et malgré les pulsions instinctives de la nature humaine}

Nous entendons ici par nature humaine les déterminations présentes chez tous les hommes indépendamment de leurs déterminations culturelles. Par ailleurs, dans Race et Histoire Lévi-Strauss montre que ce qui distingue les cultures c'est plutôt la forme que le 
contenu. Chacune d'elles a une façon particulière de résoudre des problèmes, de mettre en perspective des valeurs, qui sont quasiment pareils, «car tous les hommes sans exception possèdent un langage, des techniques, un art, des connaissances de type scientifique, des croyances religieuses, une organisation sociale, économique et politique » (50). Les éléments de base étant communs à toute l'humanité, les causes qui motivent les actions des individus doivent être à peu près les mêmes. Mais quelles sont ces causes ?

Dans la sociologie de Weber les intérêts matériels et idéels sont au centre de toutes les actions des hommes. La neurobiologie de Damasio démontre qu'elles sont déterminées par les intérêts de survie et de la qualité de cette survie. La psychanalyse de Freud témoigne que les intérêts affectifs y jouent un rôle décisif. Ces approches qui à la base paraissent divergentes en réalité convergent vers la même idée selon laquelle le principal intérêt qui guide les actions humaines est la quête du bonheur - au sens large du terme. Le problème est que maintes fois, ce qui fait le bonheur des uns ne fait pas celui des autres, de manière que cette quête devient très souvent une concurrence conflictuelle. Et la façon d'agir envers autrui dépend dans une bonne mesure de la nature de l'émotion sociale éprouvée à son égard.

Les moralistes rationalistes affirment qu'en vertu de leur faculté de rationalité les hommes privilégient plutôt les bonnes actions, les uns envers les autres. Les sciences sociales montrent que l'homme est par essence un être social. Nul ne doute que ce sont en effet les bonnes actions qui contribuent à le socialiser. Mais Freud fait douter de l'existence chez les êtres humains d'une inclination exclusive au comportement bienveillant :

La part de vérité que dissimule tout cela et qu'on nie volontiers se résume ainsi : l'homme n'est point cet être débonnaire, au cœur assoiffé d'amour dont on dit qu'il se défend quand on l'attaque, mais un être, au contraire, qui doit porter au compte de ses données instinctives une bonne somme d'agressivité. Pour lui, par conséquent, le prochain n'est pas seulement un auxiliaire et un objet sexuel possible, mais aussi un objet de tentation. L'homme est, en effet, tenté de satisfaire son besoin d'agression aux dépens de son prochain, d'exploiter son travail sans dédommagement, de l'utiliser sexuellement sans son consentement, de s'approprier de ses biens, de l'humilier, de lui infliger des souffrances, de le martyriser et de le tuer. Homo homini lupus : qui aurait le courage, en face de tous les enseignements de la vie et de l'histoire, de s'inscrire en faux contre cet adage ? (« Malaise », 65)

Le dilemme de la coexistence des sociétés humaines se situe ainsi dans le rapport entre nécessité de socialisation et désir de satisfaire les pulsions instinctives. Cela devient plus visible lorsque l'homme se met à sentir, à penser et à agir, en prenant appui sur la conscience de son âme collective. C'est une âme qui éveille en lui le sentiment de foule. Un sentiment qui produit un lien fusionnel avec son groupe d'appartenance, mais aussi stimule des 
réactions d'hostilité envers tout ce qui lui est étranger. Ceci est dû au besoin d'autoaffirmation et de distinction propres à la constitution d'une foule.

Pour Freud, la persistance dans le temps du déploiement automatique d'émotions sociales négatives s'explique par fait que malgré tout son progrès évolutif, la rationalité humaine n'a pas été capable de s'imposer aux pulsions instinctives typiquement asociales, tels que la haine et l'agressivité. Le comportement hostile envers autrui est un phénomène qui se produit également dans les micros rapports sociaux dont les relations conjugales, amicales, parentales et filiales. Mais notons qu'ici l'intensité des sentiments négatifs dirigés vers autrui est atténuée en vertu du lien personnel existant entre les parts impliquées. Lorsqu'il s'agit de macro rapports sociaux « les sentiments de répulsion et d'aversion qu'on éprouve à l'égard d'étrangers » se traduisent par «l'expression d'un égotisme, d'un narcissisme qui cherche à s'affirmer » («Psychanalyse », 37) en se dressant contre tout ce qui le contrarie, pouvant même prendre l'ampleur d'un combat sans limites.

L'éducation est sans doute un agent qui contribue à promouvoir des réactions psychiques d'ordre éthique. Mais cela ne suffit pas. L'intelligence n'est pas une force autonome, mais dépend de la vie affective. De sorte que les arguments logiques sont «sans pouvoir contre les intérêts affectifs et c'est pourquoi la lutte à coup de raisons est, dans le monde des intérêts, si stérile»(24-25). Le seul agent capable de faire face aux pulsions négatives est le lien libidinal. Sous son influence, l'individu parvient, sinon à supprimer, du moins à contrôler voire inhiber ses pulsions narcissiques au profit des pulsions affectives favorables à la sociabilité.

On nous demandera, à ce propos, si une simple association d'intérêts, sans intervention d'un élément libidinal quelconque, n'est pas de nature à comporter la tolérance réciproque et le respect pour les autres. À cette question il est facile de répondre qu'il ne peut s'agir dans ce cas d'une limitation permanente du narcissisme, car dans les associations de ce genre, la tolérance ne dure pas plus longtemps que l'avantage immédiat qu'on retire de la collaboration avec les autres. (38)

On pourrait encore objecter que l'attachement affectif constitue lui aussi un intérêt. C'est juste. Néanmoins, il est le seul intérêt capable d'opérer une restriction effective du narcissisme. Dans le cadre des relations personnelles avec parents, frères et sœurs, la personne aimée, les amis, on peut dire que le lien affectif est une conséquence naturelle en ce sens que ces relations comportent en elles-mêmes une dimension affective. Mais il ne faut pas s'attendre au même lorsqu'il s'agit des interrelations sociales entre personnes étrangères. Dans ce cas, l'établissement d'un lien affectif dépend de la mise en place d'un mécanisme 
que la psychanalyse nomme identification. Celle-ci est «la forme la plus primitive d'attachement affectif », et «peut avoir lieu chaque fois qu'une personne se découvre un trait qui lui est commun avec une autre personne, sans que celle-ci soit pour elle un objet de désirs libidineux » (42-43).

L'identification étant une condition nécessaire au lien affectif, le seul capable de freiner les pulsions hostiles envers les étrangers à notre «moi », il faut conclure que les stimulations qui font obstacle à la mise en œuvre du mécanisme d'identification ne peuvent nullement motiver des réactions psychiques d'ordre éthique. Les stimulations fondées uniquement sur des principes de différenciation mobilisent plutôt les sentiments sociaux négatifs.

Malgré l'impossibilité du projet d'une humanité harmonieuse et pacifique, si la société humaine parvient à limiter la manifestation de comportements asociaux cela représente déjà un grand pas vers une coexistence basée sur la tolérance et le respect mutuels. Dans la suite on verra que le côté positif de la nature humaine est toutefois en mesure de faire beaucoup mieux que cela.

\section{Grâce à la mobilisation de sentiments sociaux positifs}

Le témoignage psychanalytique de Freud indique non seulement le besoin mais aussi la viabilité de la stimulation des pulsions affectives positives afin de promouvoir des modes de coexistence moins nuisibles entre les êtres humains. Après tout, il est possible d'établir un lien affectif même avec des personnes qui sont étrangères à son soi sans que pour autant celles-ci soient à la fois l'objet d'un lien personnel. Si d'une part «l'autre » représente une menace pour le «moi », d'autre part «l'autre» peut aussi être pour le «moi » une source de bonheur.

Sur cette question du rôle de «l'autre » dans l'édification du « moi », la neurobiologie de Damasio donne encore une fois raison à Spinoza. Selon la théorie spinoziste, la préservation de soi et de l'autre est un moyen de renforcer le conatus de l'individu. Le conatus est l'effort de l'individu à persévérer dans son être. Ainsi, l'homme est de par sa nature enclin à chercher des formes coopératives de coexistence afin d'optimiser non seulement la garantie de survie, mais aussi la qualité de cette survie. «A l'homme rien de plus utile que l'homme ", souligne Spinoza (285). La preuve en est dans l'existence d'un décret humain, provenant d'un libre choix et non d'une détermination (loi de la nature). Au nom de ce décret, les hommes renoncent ou sont contraints à renoncer à quelque chose du droit qu'ils ont de nature et s'astreignent à une certaine règle de vie. Néanmoins, malgré la tendance humaine à chercher plutôt des formes de coexistence coopératives, l'exploitation de 
beaucoup d'émotions sociales négatives dans les cultures modernes fait que ce décret est difficile à mettre en pratique et à faire progresser.

Cela s'explique peut-être par le manque de connaissance de la valeur cognitive de l'émotion. Mais les preuves démontrant que les émotions déterminent notre façon de raisonner amènent à conclure que stimuler des émotions sociales positives n'est ni sans objet ni sans nécessité. C'est ce type de motivation que Julian Stallabrass identifie dans les effets produits par l'approche photographique de Salgado sur la condition de souffrance de $1 ' \ll$ Autre $»$ :

Selon l'argument de Kate Soper, il ne peut y avoir « extension d'empathie ou de solidarité envers les victimes de l'oppression que ne soit pas 'culpabilité' - si c'est bien le terme correct - sinon en interprétant ou en représentant leur triste condition à la lumière de notre propre identité ; c'est-à-dire, à la lumière d'une conception de ce que cela nous ferait d'être à leur place, ce que nous ressentirions si c'était à nous d'endurer leur sort ». Des photographies comme celles de Salgado se fondent justement sur un tel acte d'imagination. (32)

Les apports des recherches actuelles sur la nature de l'empathie humaine confirment la pertinence de l'argumentation de Stallabrass. L'empathie est la source de la motivation altruiste - à ne pas confondre avec la détermination -, et c'est en effet au travers de l'imagination qu'opère son mécanisme de fonctionnement. Tout cela a pu être démontré au cours de nombreuses expériences réalisées dans des domaines distincts des sciences du comportement humain. Regardons un peu plus dans les détails en quoi consiste ce type de rapport à la souffrance d'autrui, mentionné par Soper.

D'après Batson, l'empathie est la capacité de faire l'expérience et de comprendre ce que les autres ressentent sans se confondre soi-même avec autrui. Mais, cette expérience ne peut se réaliser qu'à la condition de l'existence d'une motivation qui se produit par moyen de l'intérêt empathique. Celui-ci est «orienté en direction de l'autre, en ce qu'il implique un sentiment pour l'autre. Cela inclut les sentiments de sympathie, de compassion, d'affection et de ressemblance»(8). Les émotions sociales négatives, en revanche, constituent un empêchement effectif à l'expérience de l'empathie.

Lorsque l'intérêt empathique est réveillé, nous avons le potentiel de prendre soin des autres par considération pour eux, et non simplement pour nous-mêmes. Ce qui déclenche ce sentiment est la faculté de l'imagination. Déjà au dix-huitième siècle, le philosophe et économiste Adam Smith postulait que l'imagination possède le pouvoir de nous faire nous placer dans la situation de l'autre et d'une certaine façon devenir la même personne. Postulat confirmé par les sciences sociocognitives. Par moyen d'une investigation de neuroimages 
Philip. L. Jackson, Andrew N. Meltzoff et Jean Decety ont réalisé une expérience qui a montré l'existence d'une similitude cérébrale partielle entre la perception de la douleur expérimentée en soi-même et dans un autre individu. Il a été également constaté qu'au niveau neurocognitif la perception de la souffrance sociale est analogue à celle de la douleur physique. De nombreuses autres études ont encore montré que l'empathie joue un rôle central dans le raisonnement moral, motive le comportement pro-social, et inhibe l'agression envers les autres.

En disant que son but est de faire en sorte qu'un Américain, un Français ou un Allemand qui regardent ses images arrivent à voir que les gens représentées leur ressemblent quelque part, Salgado indique, consciemment ou non, l'objectif de susciter chez ces spectateurs un intérêt empathique. On peut objecter que l'empathie se produit naturellement dans les interactions interpersonnelles des individus. Comme le fait Jean-François Chevrier en soulignant que «l'émotion est d'abord un mouvement interne aux images, plutôt que cette empathie démonstrative » de la photographie de Salgado (Le Monde, 19/04/2000). Mais en réalité, elle découle toujours d'une motivation.

Les bons résultats obtenus dans l'utilisation de la méthode de l'empathie-induite, pour motiver l'altruisme afin d'améliorer les attitudes envers des groupes stigmatisés, montrent que l'on peut faire beaucoup en vue d'améliorer les interrelations sociales des individus. Mais si l'on pense à la quantité d'images de souffrances sociales qui circulent tous les jours dans la sphère planétaire de la communication médiatique sans pour autant que cela produise des effets poussant le spectateur à «agir» pour les combattre ou les soulager, on doute du potentiel de l'image à réveiller l'intérêt empathique. Pourtant, ce qui fait défaut est la qualité et non la quantité.

L'analyse d'André Rouillé sur le rôle social de la photographie de reportage indique que ce n'est ni l'homme qui est en panne de réactivité empathique ni l'image qui serait incapable de la stimuler. Le problème se trouve dans l'actuelle politique de l'image qui donne à la photographie de reportage, particulièrement les images de la guerre, la liberté de médiatiser la souffrance, mais pas de la représenter. Après avoir cru dans la puissance de la médiatisation et pas trop dans celle de la représentation, les détenteurs du pouvoir ont tiré des leçons avec l'imagerie produite pendant la guerre du Vietnam :

Portés par l'action et confiants dans les vertus de la communication, les acteurs du nouveau système médiatique mettent, avec la photographie et la télévision, de façon inouïe les horreurs de la guerre sous les regards de tous. Mais les revers américains face à la résistance vietnamienne transforment rapidement l'adhésion des civils en émotion profonde, et suscitent des vastes mobilisations 
antimilitaristes. (179)

Dès lors les militaires et les gouvernants ont compris l'importance stratégique du discours visuel des images. Ainsi, le nouveau mot d'ordre était d'y tenir la bride haute. Les guerres des Malouines, de l'Irak contre l'Iran, et de l'Algérie, marquent de début de la fermeture du monde à l'image et en même temps la mise en place d'une politique très restrictive de l'information.

Conscients des effets que les images peuvent avoir sur les opinions publiques, les militaires ont cherché systématiquement à s'en rendre maîtres, à bannir les vues prises depuis le sol, à proscrire toute représentation de corps et de victimes, à rejeter toute trace concrète de combat, et surtout à présenter l'ensemble du conflit sous la forme désincarnée d'un immense jeu électronique. La guerre devenait ainsi abstraite et acceptable, sinon accepté. (181-182)

Dans la mesure où Salgado cherche à produire une imagerie qui incarne la souffrance représentée, on peut dire que sa photographie constitue une subversion de l'ordre établi. Sa formalisation esthétique stimule une relation de proximité avec le monde et les personnes représentées, et par là, se conforme au principe de l'empathie-induite. Pour David Levi Strauss, au travers de l'identification esthétique c'est possible de faire en sorte que les personnes différentes se reconnaissent les uns les autres (10). De sa part, Jean-François Chevrier signale en reproche que cela produit « un effet de visibilité continue, homogène, qui dissout toute opacité, toute résistance à l'image, toute altérité (ou étrangeté) » (Le Monde, 19/04/2000). Rappelons que l'objectif de Salgado est de promouvoir une économie de proximité entre les individus différents à cause et malgré les différences qui les séparent, et non de creuser davantage le fossé déjà existant. Il n'y a pas trop longtemps Freud postulait que la sympathie naissait seulement de l'identification, ce qui de nos jours est confirmé par les apports des sciences du comportement humain.

\section{Conclusion}

Du fait de privilégier une construction discursive qui met en avant l'appel aux émotions au détriment de l'argumentation « logique », Salgado est accusé par ses opposants de manque d'objectivité. Néanmoins, comme toute image, l'image photographique «a pour spécificité d'émouvoir» (Mondzain, « Commerce », 230). En outre, l'une des caractéristiques constitutives de tout discours visuel qui porte sur la thématique de la condition de vie des personnes en condition de souffrance sociale c'est l'appel aux émotions, qu'elles soient positives ou négatives de sorte que le caractère émotif de son énonciation photographique n'indique pas en soi, de manière apriorique, l'existence d'une illégitimité dans le procédé 
énonciatif de Salgado; même si à posteriori on peut, bien entendu, considérer l'émotion suscitée comme elle-même illégitime.

L'intention de Salgado n'est pas de faire comprendre la faim, la pauvreté, la misère représentées dans ses images, mais plutôt de susciter des émotions propres à éveiller l'intérêt empathique du spectateur. Ce sentiment, « grâce auquel nous découvrons la sympathie naturelle et émotionnelle qui nous lie aux autres »(Damasio, «Spinoza », 266). Ainsi, en opposition à « la pratique sociale qui consiste à simplement observer de façon distanciée et à saisir de manière instrumentale d'autres personnes » (Honneth 118), Salgado évoque dans ses images un rapport de proximité. «Tout ce qui était éloigné dans la représentation ressuscite en présence », observe Debray (Marianne, 22/03/1999).

Un professeur à l'Université de Californie témoigne des effets produits par la photographie de Salgado lorsqu'elle observe la réaction du public qui a visité l'exposition du photographe, au Musée d'Art de Berkeley. «On devrait visiter cela deux fois, dit Candace Slater, - une pour regarder les photos et l'autre pour regarder le visage des gens en regardant les photos. [...] Vous pouvez voir dans leurs visages colère, effroi et incompréhension ${ }^{2}{ }^{2} \mathrm{De}$ tels effets ne se produisent pas de manière accidentelle mais en vertu de l'effort de Salgado à composer des images qui ne soient pas des rapports «cliniques» de la triste condition des personnes affectées par les souffrances sociales. «Il faut aller à fond, dit-il, aider à sentir le monde. C'est cela qui est capital » (Jornal de Fotografia, 09/06/1995).

Les critiques qui s'opposent à l'approche photographique de Salgado indiquent que l'appel aux émotions « dépolitise » les spectateurs. N'oublions pas en tout cas qu'en vertu de sa valeur cognitive, l'émotion a plus affaire avec la politique qu'on ne le dit.

\section{Bibliographie}

Aumont, Jacques. L'image. Paris : Nathan, 2000.

Barthes, Roland. La chambre claire : note sur la photographie. Paris : Seuil, 1980.

Batson, Daniel Charles. «Empathy-Induced Altruistic Motivation ». Herzliya Symposium on Prosocial Motive : Emotions and Behavior, 2008.

Chevrier, Jean-François. « Salgado, ou l'exploitation de la compassion ». Le Monde, 19 avril 2000.

Damasio, Antonio. Spinoza avait raison. Paris : Odile Jacob, 2005.

---. L'erreur de Descartes. Paris : Odile Jacob, 2006.

Debray, Régis. « Exodes ». Marianne, 22 mars1999.

Freud, Sigmund. Malaise dans la civilisation. Paris : PUF, 1971. 
---. Essais de Psychanalyse. Paris : Payot, 1981.

Guerrin, Michel. «Une iconographie religieuse ». Le Monde, 11 avril 2000.

Honneth, Axel. La réification : petit traité de théorie critique. Paris : Gallimard, 2007.

Jackson, Philip. L., Andrew N. Meltzoff, Andrew et Jean Decety. «How do We Perceive the Pain of Others? A Window into the Neural Process Involved in Empathy ». Elsevier, 14 november 2004.

Levi-Strauss, Claude. Race et Histoire. Paris : Gallimard, 1987.

Mondzain, Marie-José. L’image peut-elle tuer ? Paris : Bayard, 2002.

---. Le commerce des regards. Paris : Seuil, 2003.

Roth, Gehard. « Die Ratio allein bewegt überhaupt nichts ». Der Spiegel Wissen, 1/2009.

Rouille, André. La photographie. Paris : Gallimard, 2005.

Sischy, Ingrid. «Good Intentions ». The New Yorker, 09 septembre 1991.

Spinoza, Baruch. Éthique. Paris : Gallimard, 2009.

Stallabrass, Julian. «Sebastião Salgado and Fine Art Photojournalism ». New Left Review, May-June, 1997.

Strauss, David. L. Between the Eyes : Essays on photography and politics. New York : Aperture, 2003.

Tisseron, Serge. Y a-t-il un pilote dans l'image? Paris : Aubier, 1998.

NOTES

1 On entend ici par normal, les enfants qui ne sont pas atteints d'autisme.

2 «Epic Sebastião Salgado show at the Berkeley Art http://www.berkeley.edu/news/media/releases/2002/01/18_salgado.html (consulté le 16 mars 2007). 